\title{
A Sensitivity Investigation on the Aeroelastic Dynamic Stability of Slender Spinning Sounding Rockets
}

\author{
Roberto Gil Annes da Silva, José Guido Damilano¹, João Luiz F. Azevedo
}

\begin{abstract}
The present work addresses a sensitivity analysis investigation of the aeroelastic stability margins for the VSB-30 sounding rocket during the atmospheric flight phase. Parametric stability analyses are performed considering variations of the inertia properties of the modular payload. Such variations can be caused by different type and/or number of experiments (payload modules). The aerodynamic model is based on a supersonic unsteady potential aerodynamic method. Freestream conditions depend on the flight speed and atmosphere. An equivalent structural dynamic model of the rocket is represented by a beam-like structure. The objective of this investigation is to establish an aeroelastic model for aeroelastic stability and response analyses, as well as a procedure for the identification of stability margins for rockets. The resulting aeroelastic model should be further used in MDO processes for the improvement of the vehicle flight performance. The results of the present effort indicate that the flutter behavior of the VSB-30 sounding rocket is sufficiently robust inside the operational envelope, even considering the environmental and loading conditions. The spinning effect, in this case, does not play a significant role, because the flutter margins remain almost unaltered with and without VSB-30 body spin.
\end{abstract}

KEYWORDS: Sounding rockets, Aeroelastic analysis, Flutter margins, Sensitivity studies.

\section{INTRODUCTION}

European research on microgravity required a new sounding rocket with performance similar to the one delivered by the English rocket Skylark-7, whose production had been discontinued. Instituto de Aeronáutica e Espaço (IAE), in Brazil, took on the task of the rocket development and complete integration in a joint effort with the Deutsches Zentrum für Luft und Raumfahrt (DLR), responsible for the payload of microgravity experiments. Several modifications were performed on the previously developed VS-30 sounding rocket, in order to satisfy the required specifications for new scientific andtechnologicalexperimentsinthemicrogravityenvironment. The resulting modified vehicle, named VSB-30, is a two-stage spinning-stabilized slender sounding rocket, with two sets of three fins on each stage, whose engines use a solid propellant (Duarte et al., 2005).

The VSB-30 rocket flight operation is divided into two phases: the first stage flight (FSF) and the second stage flight (SSF). The engines accelerate the vehicle to a ballistic flight path towards the desired microgravity condition. A more recent application of the baseline VSB-30 sounding rocket configuration regards in-flight experiments on the aerodynamic behavior and thermal problems of an unconventional asymmetric shape for reentry vehicles comprising multifaceted surfaces with sharp edges (SHEFEX) (Turner et al., 2006). The aim of the experiment was to correlate numerical analysis results with actual flight data regarding the aerodynamic effects and structural concept for the thermal protection system. Hence, the development of an aeroelastic model of the VSB-30 vehicle should be very useful 
for the aeroelastic stability and response investigation for any type of proposed configurations, including SHEFEX.

Typically, slender-body finned vehicles can present two types of flutter mechanisms, i.e., fin bending-torsion flutter and body pitch-bending flutter (Martin, 1958). Examples of rocket flutter analysis have been well documented in several reports and articles (Azevedo, 1988; Bae et al., 2004; Francesco Capri et al., 2006; Garcia-Fogeda and Liu, 1988; McNamara and Friedmann, 2006; Paek et al., 2002). Some of them concern fin flutter, for example, when the rocket presents deployable fins. Nonlinear phenomena, such as free-play, also lead to nonlinear aeroelastic behavior (Bae et al., 2004).

In the literature, several efforts have been well documented (Chae, 2004; Chae and Hodges, 2003; Haddadpour, 2006; Heddadj and Cayzac, 2000; Livshits et al., 1996; Meyers, 1973; Murphy and Mermagen, 2001; Murphy and Mermagen, 2005; Platus, 1992), regarding the investigation of the flight dynamic/aeroelastic response behavior of free flight spinning (or not) rockets and missiles. Moreover, in some studies the coupling between flight mechanics, aeroelasticity, and control system (aeroservoelastic coupling) has been investigated (Haddadpour, 2006). Motivations for these studies are usually associated to the fact that flight vehicles with slender configurations might experience aeroelastic instability during flight due to coupling between a short period rigid-body mode and a body-bending mode. Such a coupling might affect the planned trajectory of the vehicle and, in the case of military rockets, the weapon aiming system could be compromised.

Most of the previously cited investigations employ slender body theory for the aerodynamic model of the elastic body, combined with a quasi-steady aerodynamic model representing the body global aerodynamic coefficients. However, for the best assessment of aeroelastic instabilities, a more accurate prediction method to compute the unsteady aerodynamics was presented by Garcia-Fogeda and Liu (1988), the so-called Harmonic Potential Panel (HPP) method. Further development on more accurate methods, based on lifting surface theory, for the prediction of unsteady airloads aeroelastic to analyze supersonic wingbody configurations were presented by Chen and Liu (1990), and Liu et al. (1997), which is, in fact, a unified hypersonicsupersonic lifting surface method.

Nevertheless, lifting surface methods are limited to linear computation of the unsteady airloads. For this reason, computational fluid dynamics for unsteady aerodynamic modeling of transonic airloads has also been used for transonic aeroelastic analysis of body alone rockets (Azevedo, 1988) and wing-body-like vehicles (Francesco Capri et al., 2006). A review of the state-of-the-art on advanced methods for computational fluid dynamics and heat transfer applications of hypersonic aeroelasticity and aerothermoelasticity is presented by MacNamara and Friedmann (2006).

The scope of the present investigation is the study of the aeroelastic stability (flutter) of a free flight spinning rocket. The strategy for the parametric investigation regarding a sensitivity analysis should be based on the identification of the flutter margins as a function of the variation of the inertial properties of the payload, solid propellant consumption, as well as the flight environmental conditions. A set of adequate steps for an aeroelastic investigation of sounding rockets is proposed. The procedure for the analysis, as well as the assumed hypotheses, should be mainly based on the flight dynamic behavior of the vehicle during its operation. It is important to note that differently from conventional aircraft, the rocket atmospheric flight does not present a steady behavior as in a cruise condition. The vehicle should be subjected to strong environmental variations associated to its flight level, flow speed and its inertial characteristics.

\section{MATHEMATICAL MODELS}

\section{Structural Dynamic Model}

The VSB-30 airframe is represented by a finite element structural dynamic reduced order model, composed by beam and plate elements connected by nodes to represent the body, the fin spars and the fin surfaces, respectively. Such an approach is sufficient for the aeroelastic stability analysis of the vehicle. In the vehicle body nodes, the distribution of inertial characteristics is approximated by lumped masses representing the inertia properties. A sketch of the finite element model, including the distributed masses, will be presented in the forthcoming paragraphs. Since the rocket engines burn the solid propellant, a considerable rate of change in mass should be considered in the modeling of the vehicle structure. Thus, mass properties represented in the structural dynamic model, for each flight condition, change as a function of time.

Furthermore, depending on the payload configuration, there is also a significant change in mass to be considered for the structural dynamic modeling. The structural 
model was developed with the MSC/NASTRAN ${ }^{\circledR}$ software (MSC/NASTRAN, 1995) to compute the natural frequencies and mode shapes for the analysis, assuming a piecewise linear model, because the mass variation should be assumed as constant at a given instant.

The stability of the VSB-30 rocket is guaranteed by the fins and the induced spin after liftoff. Therefore, it would be desirable to include spinning effects in the structural dynamic modeling. Spinning effects can lead to changes on mode shapes and natural frequencies of structures. Paek et al. (2002) presented results on the investigation of wraparound finned projectiles flutter. Results of their aeroelastic analysis indicate that flutter speeds depend on the roll rate. Furthermore, in that special case, there was a significant deformation of the wrapped fin with the increase of the roll rate. For their analysis the order of the roll rate was about three times the first natural frequency of the body and ten times smaller than the first natural frequency of the fin (1st fin bending). So, it was possible to conclude that the spinning effects had to be taken into account for the identification of the rocket mode shapes and natural frequencies.

Figure 1 presents the roll rate for the VSB-30 vehicle until 40 seconds of flight. It is observed that the rolling frequencies are moderately low. Therefore, such an effect will not be considered in the VSB-30 rocket analysis because the spinning rates are between four to six times smaller than the first mode shape of the vehicle (body 1st bending), thus not inducing significant changes in stiffness nor in gyroscopic effects.

On the other hand, even in this case, an investigation on the spinning effects was performed, supposing that this

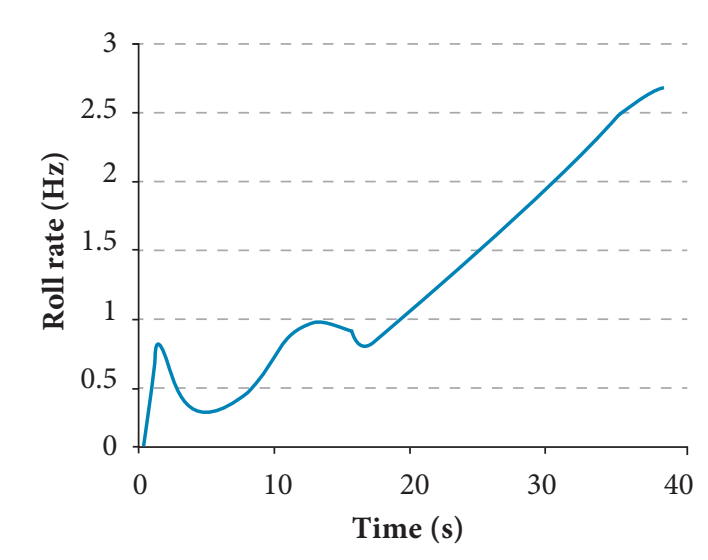

Figure 1. Roll rates of the VSB-30 vehicle after liftoff. aeroelastic analysis methodology could be applicable to other classes of sounding rockets. From the dynamics rotating system, it is desirable to consider spinning effects, which might include the Coriolis and centrifugal effects by introducing the gyroscopic damping matrix into the dynamic equations, as well as the differential stiffness due to nonlinear effects (Heddadj and Cayzac, 2000). These effects are significant when the spinning roll rates are larger than the current ones (Fig. 1). The inclusion of such effects may lead to structural dynamic changes that might influence the flutter mechanisms of the airframe. A MSC/NASTRAN DMAP option was used to compute the modal characteristics of the rotating vehicle, and the corresponding theory is well documented in MSC/NASTRAN V70 (1995).

\section{Unsteady Aerodynamic Model}

In the present investigation, only aeroelastic analyses at supersonic flow conditions will be performed. The aerodynamic modeling method of unsteady linear potential flows is based on the discrete kernel function approach. The development of discrete element kernel function methods is based on integral solutions of the small disturbances linearized potential flow equation. In this work, the ZONA7U method (ZAERO, 2003), implemented in the ZAERO ${ }^{\circledR}$ software system, has been used to model the unsteady airloads for flutter analysis (Liu et al., 1997). Chen and Liu (1990) present the mathematical formulation of the ZONA7 method for supersonic aerodynamic modeling of wing body configurations. Its extension, which accounts for the lifting surface thickness effects, is named ZONA7U method (Liu et al., 1997), and is also implemented in the ZAERO ${ }^{\circledR}$ software package.

The ZONA7U method is different from ZONA7 due to the inclusion, in the former, of the thickness effect correction. Thickness corrections are an important issue to be considered, leading to the improvement of the flutter speed prediction (Liu et al., 1997). In most cases, the flutter speed decreases when the fin thickness is considered (Liu et al., 1997).

Special care should be taken during the meshing process. For example, depending on the Mach number, the lifting surface elements (panels) should have an adequate aspect ratio for the computation of supersonic flows. Another important issue to be considered is the need for a mesh convergence study based on the resulting flutter speed dispersion. 
Such study might be seen as a tool to identify the best costbenefit relation regarding the size of the aerodynamic mesh for modeling the body and lifting surfaces unsteady aerodynamics.

\section{AEROELASTIC MODELING}

The scope of the aeroelastic analysis to be presented here is the flutter investigation of a spinning sounding rocket based on the unsteady aerodynamic model of the full vehicle. It is usual to investigate rocket fin flutter by just modeling the isolated fin (Bae et al., 2004; Paek et al., 2002). However, possible interference effects could influence the unsteady aerodynamic behavior of the vehicle, and, for this reason, the full vehicle should be modeled. The structural dynamic behavior is also assumed as linear, and the spinning effect is neglected for the aforementioned reasons.

It is supposed, as a first approach, that the present aeroelastic model is linear regarding the aerodynamic and structural models. The scope here is the investigation of the vehicle flutter mechanisms. Small aeroelastic displacements are assumed and the development of this baseline aeroelastic model will be important for further studies on Multidisciplinary Design and Optimization (MDO) applied to the vehicle design and upgrade processes. Also, this baseline linear model should be important for future development on computational aeroelasticity applied to transonic aeroelastic stability and response, assuming nonlinear aerodynamic models for correction of the linear aerodynamic methods (ZAERO, 2003).

\section{Description of the Vehicle}

The VSB-30 sounding rocket is composed of two tandem rocket engines. Its total length is $12.699 \mathrm{~m}$ and it has a total mass of about 2,200 kg at liftoff. Evidently, this may vary depending on the payload configuration. In the present study, only the aeroelastic analysis of the VSB-30 sounding rocket assembled with modular cylindrical payload modules is investigated. These modular payloads are assembled in tandem as a set of two, three or four modules. They are mass balanced in relation to the vehicles longitudinal axis (assumed here as the " $\mathrm{x}$ " axis). Figure 2 presents the vehicle finite element structural model and the corresponding CAD drawing.

\section{Basic Hypotheses and Assumptions}

The VSB-30 vehicle is a ballistic sounding rocket whose flight altitude range from sea level is $250 \mathrm{~km}$. Its flight path is designed in order to achieve sufficient time in microgravity conditions for scientific and technological experiments. The vehicle reentry flight phase is not included in the scope of the present investigation. Figure 3 shows the typical altitude, Mach number, dynamic pressure and angle of attack for this class of vehicles as a function of time. The data shown represent the case for which the vehicle comprises four payload modules for scientific experiments.

Information presented in Fig. 3 allows the formulation of hypotheses and conditions to set the aeroelastic analysis procedure. One should observe that the Mach number varies up to the hypersonic flow condition, remaining constant afterwards. However, while the vehicle flies within the atmosphere, the maximum Mach number is supersonic (3.313) at the maximum dynamic pressure, which can be obtained from the corresponding chart in Fig. 3.

In fact, one can observe that there are two dynamic pressure peaks. Furthermore, each of these peaks is associated with the vehicle configuration in each stage: in the first stage, when the vehicle remains with two tandem rocket engines

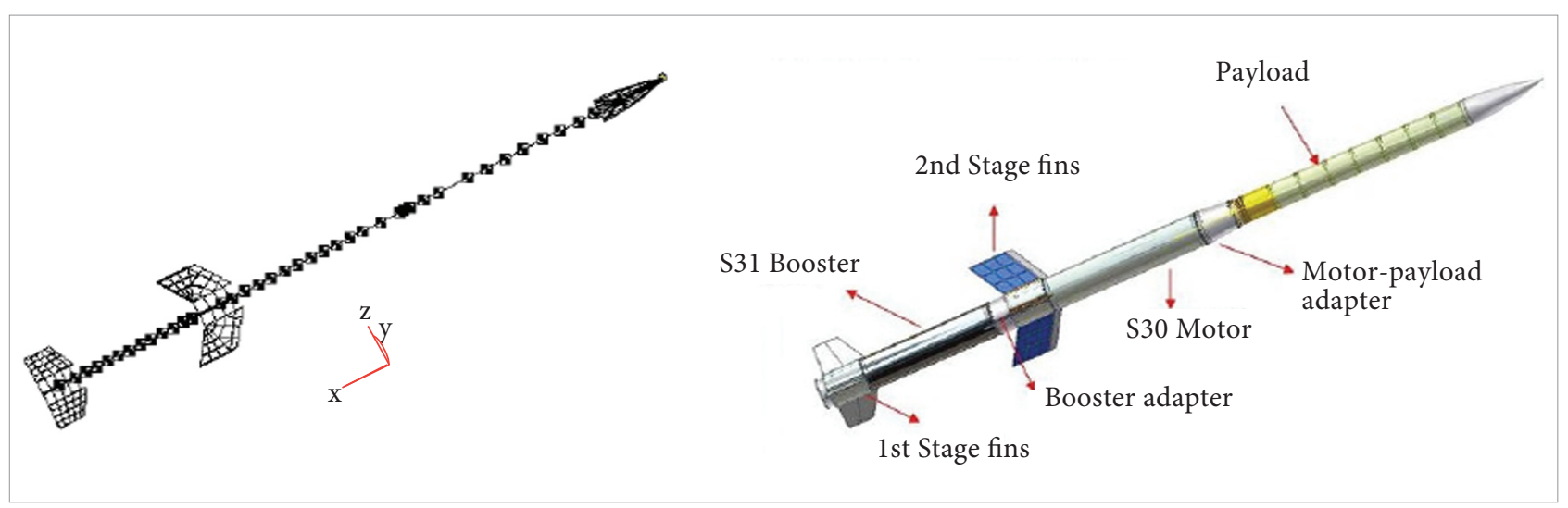

Figure 2. VSB-30 rocket: the finite element beam-plate model and the CAD drawing. 


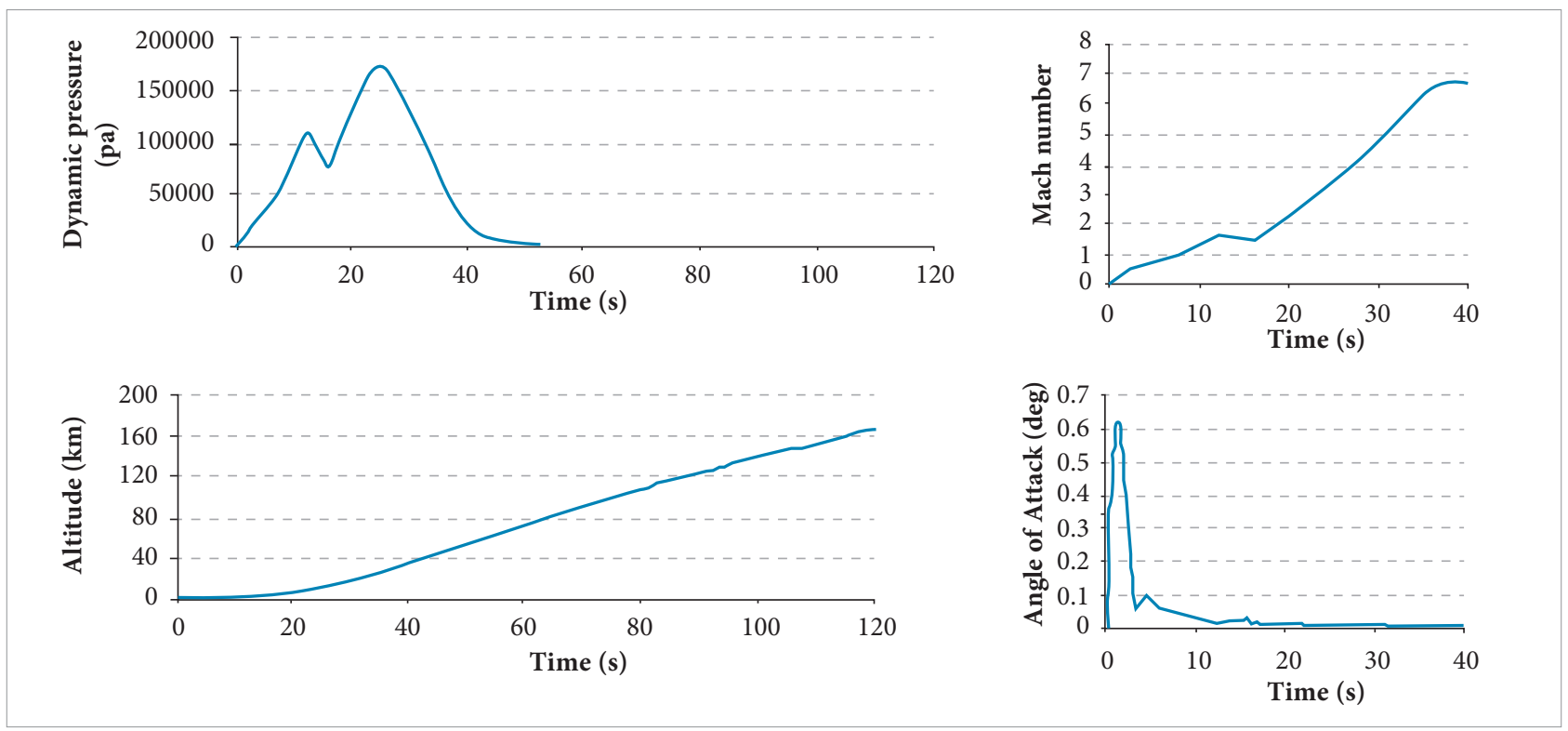

Figure 3. Typical time history curves describing the performance of the VSB-30 vehicle.

and two set of fins, and, in the second stage, in which the vehicle is composed by a single rocket engine and a single set of fins. This is the reason why the aeroelastic analysis procedure should be divided into first and second stage flight analyses. Since the stages separate, it will be necessary to consider two distinct dynamic models of the airframe, one before separation and another after separation.

In the case of a non matched-point flutter analysis, parametric variations around the maximum dynamic pressure condition are assumed. These parametric variations should be set as freestream speed and Mach number, flight altitude and mass variations regarding the solid propellant consumption and number of payload modules. This first approach for the aeroelastic analysis provides the sensitivity of the aeroelastic stability due to payload and solid propellant variations.

The spinning behavior of the vehicle is associated with the body roll rate. The spin may promote Coriolis and Magnus effects. Such effects could have an important influence on the flight dynamic behavior of the vehicle. The idea of inducing a spin during flight is related to improving the flight dynamic longitudinal stability margins of the vehicle. The reader should keep in mind that a sounding rocket is essentially a ballistic body, that is, there are no controls acting during its flight path.

Coriolis effects are associated to gyroscopic forces which result from the spin. For this reason, the structural dynamic behavior of the vehicle should be altered by the appearance of a gyroscopic damping matrix and a differential stiffness due to centrifugal forces (Francesco Capri et al., 2006). However, this type of effect can be negligible when the vehicle roll rates are small. For the present investigation, it is assumed that roll rates of $1.42 \mathrm{~Hz}$ and $0.91 \mathrm{~Hz}$ at the first and second stages, respectively, are sufficiently small and can be neglected, since they are not able to promote significant changes in the structural dynamic behavior of the vehicle.

Another effect associated to a spinning body is the Magnus effect. This type of effect is represented by a lift force induced by the rotating body in angle of attack. This effect changes the mean flow around the vehicle, and it is significant when the angle of attack is high. Thus, the small disturbance flow should not be altered by the loadingchangesaroundameansteadyflowcondition. Furthermore, from the corresponding chart in Fig. 3 , it is possible to observe that the angle of attack is sufficiently small throughout the flight path, resulting in negligible Magnus effect.

\section{Structural Dynamic Model}

The VSB-30 rocket is represented by a beam-like equivalent structure, sufficiently accurate to capture the global mode shapes of the airframe. Moreover, the fittings are represented by a set of constraints which might represent the physical coupling between the parts of the vehicle. The fins are represented by a combination of beams and plate elements, as in the real structure. The second stage engine fin internal structure resembles a "spider web" beam structure, as observed in Fig. 4. 

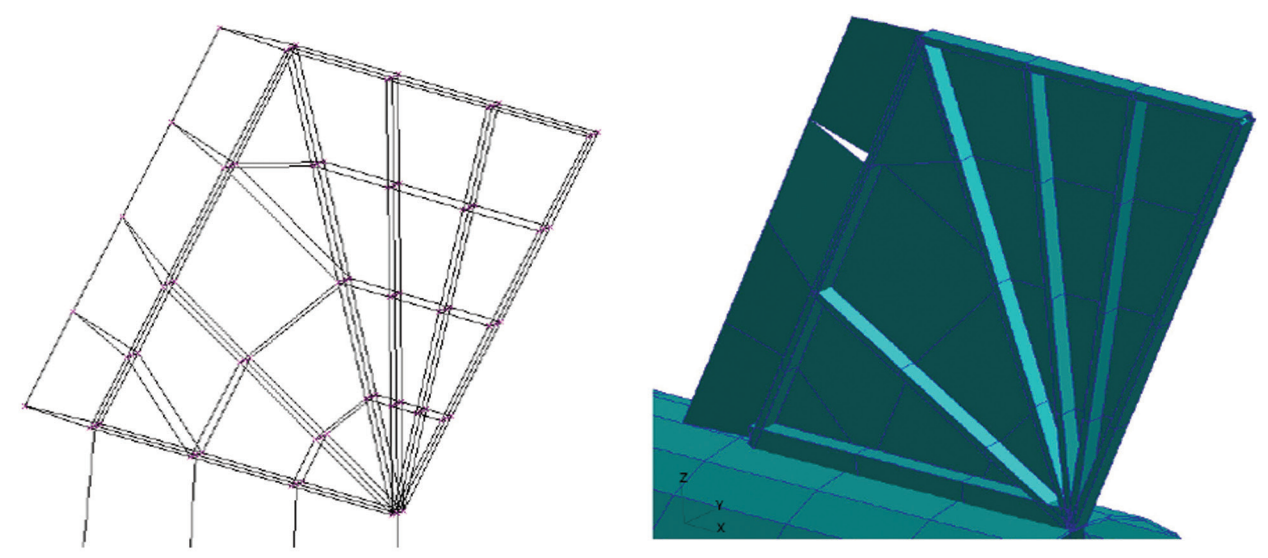

Figure 4. Finite element model of the "spider web" like structure of the second stage fins.

The mass of the solid propellant is distributed in several points along the vehicle longitudinal axis, and its consumption is represented by subtracting their corresponding value, as the propellant is burned, for each instant of analysis.

\section{Aerodynamic Model}

The aerodynamic model is developed using the ZAERO software (Chen, 1999). A body of revolution subdivided into panels was assumed to represent the aerodynamic shape of the vehicle. For the fins, a flat plate aerodynamic representation is assumed, including the thickness correction (ZAERO, 2003). The correction for thickness effects is important for Mach numbers higher than 1.2. The reader should remember that the aeroelastic stability behavior is less conservative when this correction is not considered (ZAERO, 2003).

Figure 5 shows the aerodynamic meshes used for the present investigation. Each payload configuration is represented by a proper aerodynamic mesh. Furthermore, for the first stage flight phase, the aerodynamic model represents the whole vehicle including the two sets of fins. The chosen Mach numbers for the stability investigation are the corresponding values at maximum dynamic pressure conditions. Table 1 shows the main flight parameters for each configuration and flight phases.

The maximum dynamic pressure identified during the flight of the first stage occurs when the flight altitude is around 3,400 m, at Mach number 1.6, that is, 12.5 seconds after liftoff, for the case of the vehicle configured with two payload modules. These conditions are taken as reference to perform the aeroelastic stability analyses. They are different from other configurations of the vehicle, since they also depend on the number of payload modules. It is assumed that the Mach number and flight altitude, for the reference conditions, are constant, but the freestream speed varies around the specified Mach number. Table 1 shows the flight parameters for each of the configurations analyzed, as well as the fight phases represented as "first" and "second" stages.

The number of panels, used in the aerodynamic mesh for the body, depends on the configuration to be

Table 1. Reference conditions for the aeroelastic stability analyses.

\begin{tabular}{|l|c|c|c|c|c|c|c|c|}
\hline \multirow{2}{*}{$\begin{array}{l}\text { Configuration } \\
\text { flight phase }\end{array}$} & \multicolumn{2}{|c|}{ Time (s) } & \multicolumn{2}{c|}{ Dyn. Pressure (Pa) } & \multicolumn{2}{c|}{ Mach number } & \multicolumn{2}{c|}{ Altitude (m) } \\
\cline { 2 - 11 } & 1st stage & 2nd stage & 1st stage & 2nd stage & 1 st stage & 2nd stage & 1st stage & 2nd stage \\
\hline 2 modules & 12.5 & 24.2 & 117420.0 & 179525.5 & 1.599 & 3.375 & 3518 & 11050 \\
\hline 3 modules & 12.5 & 24.5 & 112684.4 & 175899.4 & 1.558 & 3.337 & 3434 & 11037 \\
\hline 4 modules & 12.5 & 24.8 & 108724.7 & 172895.0 & 1.523 & 3.313 & 3363 & 11062 \\
\hline
\end{tabular}



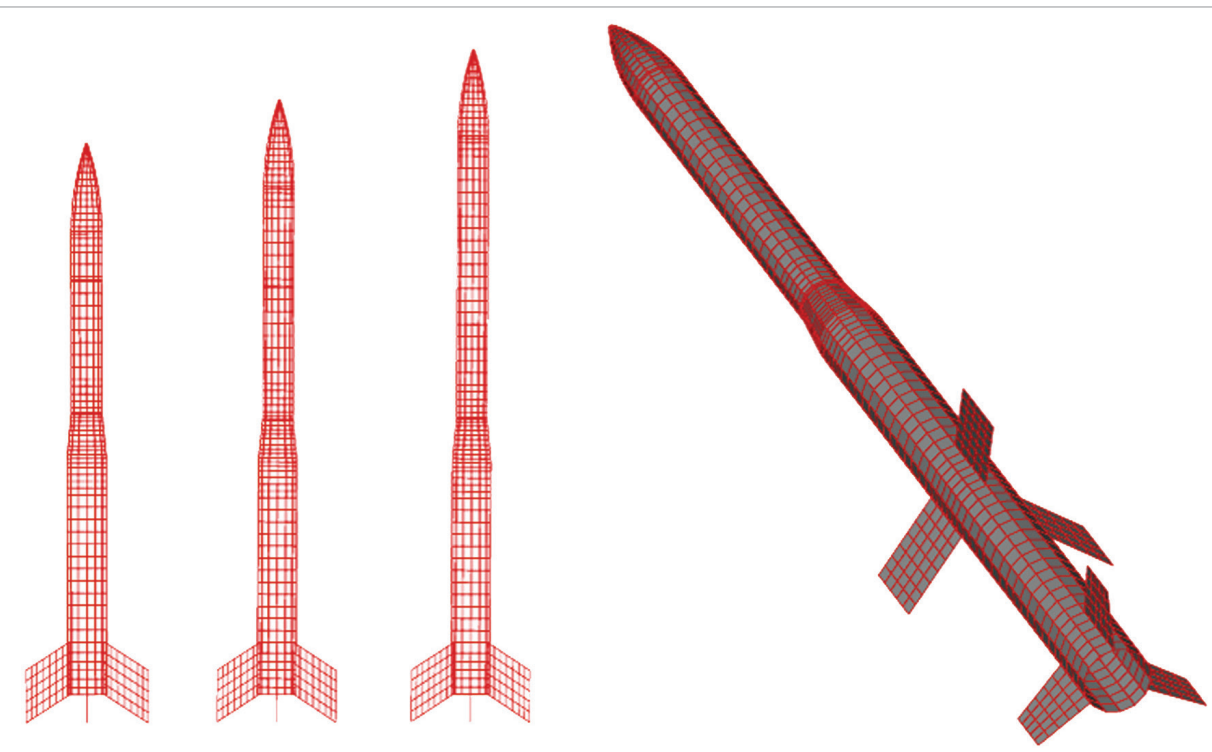

Figure 5. Aerodynamic meshes for the VSB-30 rocket for two stages configuration and four modules (right), and the single second stage for 2 to 4 modules (left).

analyzed. However, the number of panels, used to mesh the fins, remains constant since these structures do not change according to the configurations to be analyzed. The remaining task to proceed in the flutter analysis is a mesh convergence study to evaluate the robustness of the aerodynamic model for stability investigations. The idea is to identify which are the flutter modes and to proceed with the mesh refinement only in the parts of the vehicle in which modal displacements contribute for the flutter mode. Such investigations will be discussed further, as soon as the flutter mechanisms are identified.

\section{FLUTTER ANALYSIS RESULTS}

The aeroelastic analysis procedure is based on the investigation of the aeroelastic stability within the vehicle flight envelope. The strategy adopted here is to observe the dynamic pressure-time history, demonstrated in Fig. 6. In this figure, the red line indicates that the points below it may not be included in the aeroelastic analysis procedure. This is because this decrement in the dynamic pressure does not offer any risk in terms of flutter, since larger dynamic pressures are reached before, as shown in Fig. 6.

The non-matched point flutter analysis will be based on the variation of the freestream speed at each of the maximum dynamic pressure peaks, each one associated to the first and second stage flight phases. This is not the best approach for flutter substantiation since only a single eigenvalue will

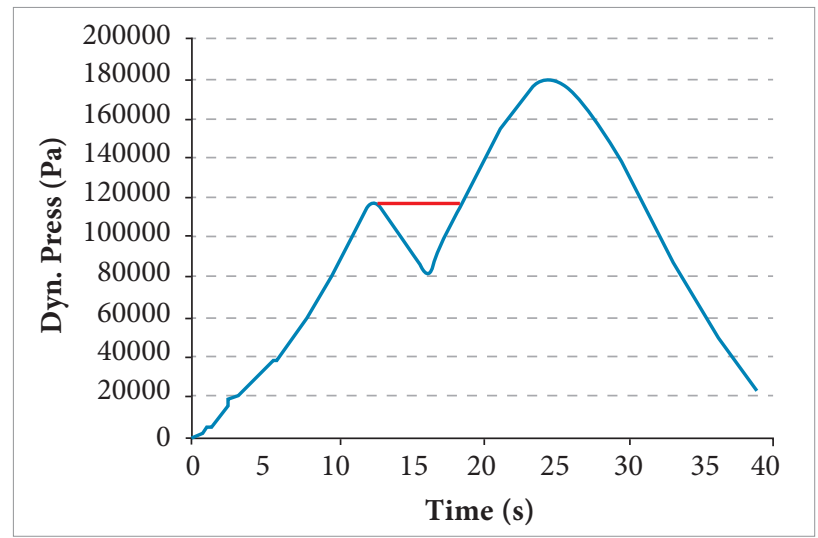

Figure 6. Typical dynamic pressure-time history.

represent the true aerodynamic damping (Chen, 1999) and the associated aeroelastic frequency. Nevertheless, this approach is fast enough to qualitatively identify the flutter mechanism to be explored on a subsequent matchedpoint flutter analysis. The reference Mach number and density are the same values identified at the maximum dynamic pressure condition.

The resulting flutter mechanism identified by the nonmatched point flutter analysis is represented by the coupling of the 7 th and 9th anti-symmetric modes. These modes are the second stage fin bending and torsion modes, which are illustrated in Fig. 7.

It is important to note that a possible introduction of the spinning effect should be responsible for altering the 
structural dynamic behavior. The flutter mechanism could be anticipated or delayed, depending on the coalescent modes. For this reason, further investigation is recommended in order to introduce the spinning effect in the vehicle dynamic model, due to the influence of the gyroscopic effects produced by the spin.

The results of the sensitivity investigation based on the number of payload modules are summarized in Table 2.

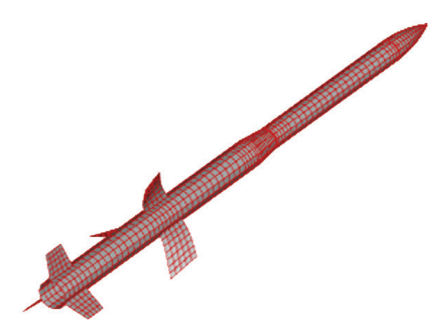

$7^{\text {th }}$ mode $[37.2 \mathrm{~Hz}]$

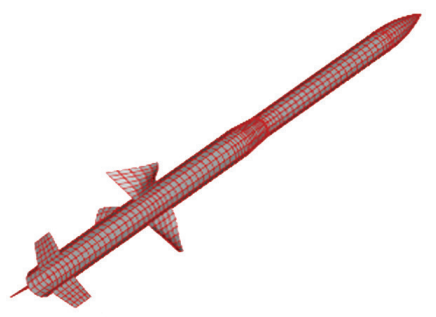

$9^{\text {th }}$ mode $[66.9 \mathrm{~Hz}$

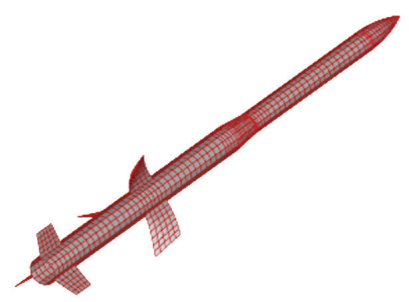

Flutter mode $(39.7 \mathrm{~Hz})$

Figure 7. Flutter mechanism in the first stage flight phase.
The reader may observe that the computed flutter speeds associated with the number of modules are related to the same flutter mechanism, and the flutter speed reduction is less that $4 \%$. This fact is a good indication that the vehicle is sufficiently robust in terms of the variations of payload mass regarding the aeroelastic stability. Moreover, this result makes sense because the flutter mode results from a coalescence of two anti-symmetric fin mode shapes. That is, the dynamic contributions of the vehicle body modes do not play an important role in the flutter mechanism.

From the discussion above, it is possible to conclude that this change in flutter speed should be mostly related to the vehicle environmental conditions, since the dynamic pressure for each of the configurations is different. Figure 8 shows a comparison between the dynamic pressures at the same time frame for two, three and four payload modules.

The most critical configurations occur when the vehicle flies with two payload modules. Such configuration, during the atmospheric flight phase, subjects the vehicle to dynamic pressures higher than the others do.

The next step in the study was an analysis still based on a nonmatched point flutter solution. However, unlike the previous procedure, this one was repeated for different Mach numbers, each of which associated to a different flight condition. In this case for the unsteady aerodynamic model used to compute the flutter condition, these reference Mach numbers are used to

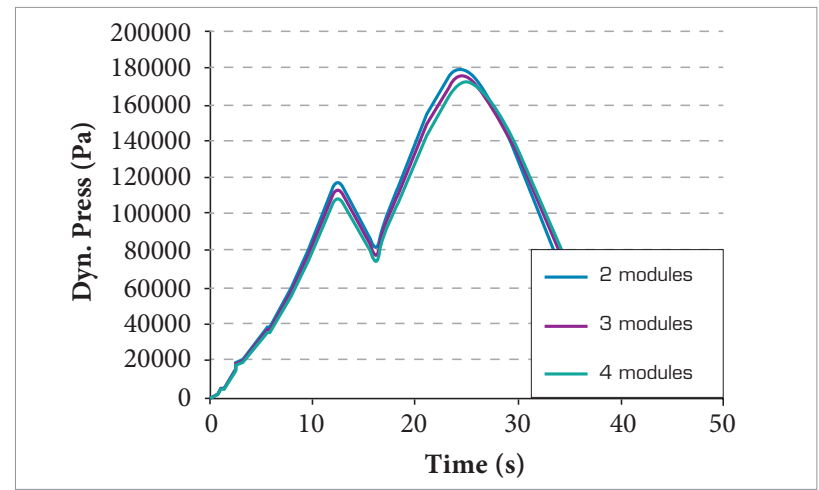

Figure 8. Comparison of the dynamic pressure time history for two, three and four payload modules.

Table 2. Flutter speeds and dynamic pressure for the VSB-30 vehicle in the first stage of flight - non-matched point flutter analysis. Number of modules 2

Flutter mode

7th and 9th

556.4935

40.0591

$1.355 \mathrm{E}+05$

\section{3}

4

7th and 9th

536.4167

550.0458

39.6766

39.8965

$1.259 \mathrm{E}+05$ 
generate the aerodynamic influence coefficients. Figure 9 shows the computed flutter dynamic pressures, when the vehicle is configured with two payload modules.

The results represented in Fig. 9 indicate that flutter dynamic pressures are above the dynamic pressure imposed on the vehicle during actual flight. It is possible to conclude, based on the studied trajectories, that increasing the number of payload modules reduces the chance for flutter, because the higher vehicle masses will decrease the flight speeds in lower altitudes.

In the second stage flight, environmental conditions are more favorable because the higher altitudes lead to lower densities and dynamic pressures. On the other hand, flight speeds are higher, approaching two times the Mach number of the first stage flight. For this analysis, the mass ratio concept is introduced to help understand why at higher dynamic pressures the same airframe remains stable in terms of flutter, as demonstrated in Table 3.

A lower mass ratio leads to a lower amount of energy needed to promote the coalescence of aeroelastic modes which may lead to flutter. On the other hand, there is a need for high kinetic energy flow from which energy would be extracted to

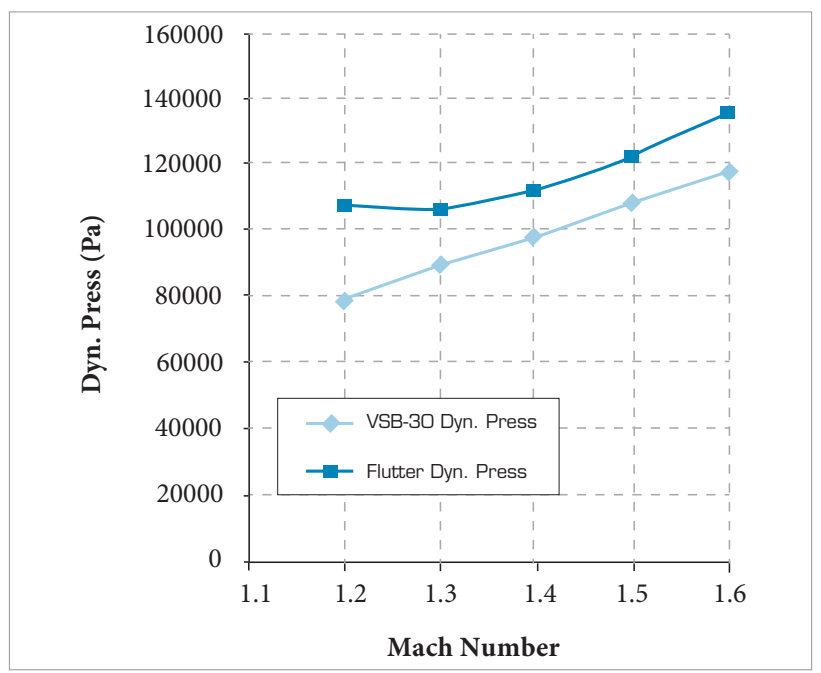

Figure 9. Flutter dynamic pressures for VSB-30 rocket, 1st stage flight, two payload modules. cause flutter. Looking at Fig. 10, it is possible to understand why, at higher altitudes and Mach numbers, even though the dynamic pressure is high, the mass ratio is also high enough, thus retarding the coalescence of the modes for flutter. This explains why it should be necessary to concentrate efforts on analyzing flutter stability nearby transonic Mach numbers.

The matched-point flutter analysis considering the pair density (altitude)/Mach number is the subsequent step in the whole process. Thus, the true aeroelastic damping curves, as functions of the freestream flow conditions, are computed. This kind of analysis is more expensive, computationally speaking, since it is necessary to calculate an aerodynamic influence coefficient matrix for each Mach number included in the profile to be investigated. Once these matrices are generated, it is possible to repeat the flutter computation for a set of Mach-altitude (density) pairs.

The results of the matched-point flutter analysis indicate that the vehicle is free from flutter mechanisms inside its flight envelope. However, the critical configuration, in terms of flutter, is the one in which the vehicle is configured with two payload modules. Figure 11 shows that in the typical matched-point flutter analysis it is likely that the 7th mode is unstable, even though its corresponding natural frequency remains practically constant.

The inclusion of spinning effects considers the vehicle rotating between $0.5 \mathrm{~Hz}$ to $10 \mathrm{~Hz}$. In the latter, the roll rates are beyond those at the maximum dynamic pressure condition during first stage flight. Here, the same conditions described above, i.e., vehicle with two payload modules, are investigated regarding the sensitivity of the flutter dynamic pressures due to a projected increase in roll rates. Figure 12 shows the sensitivity of the flutter dynamic pressure as a function of the roll rate. This result indicates that increasing the roll rate leads to a decrease in the flutter dynamic pressures, in the case of the coupling of the 7th and 9th modes. The reader should remember that these results refer to a non-matched flutter point analysis.

Looking at Fig. 13, this behavior is clearly understood. Damping and frequency evolution, as the roll rates increase,

Table 3. Flutter speeds and dynamic pressure for the VSB-30 vehicle during the second stage flight.

\begin{tabular}{|l|c|c|c|}
\hline Number of modules & 2 & 3 & 4 \\
\hline Flutter mode & 5 th & No flutter & No flutter \\
\hline Flutter speed $(\mathrm{m} / \mathrm{s})$ & 1950.773 & No flutter & No flutter \\
\hline Flutter frequency $(\mathrm{Hz})$ & 54.1746 & No flutter & No flutter \\
\hline Dynamic pressure $(\mathrm{Pa})$ & $7.376 \mathrm{E}+05$ & No flutter & No flutter \\
\hline
\end{tabular}




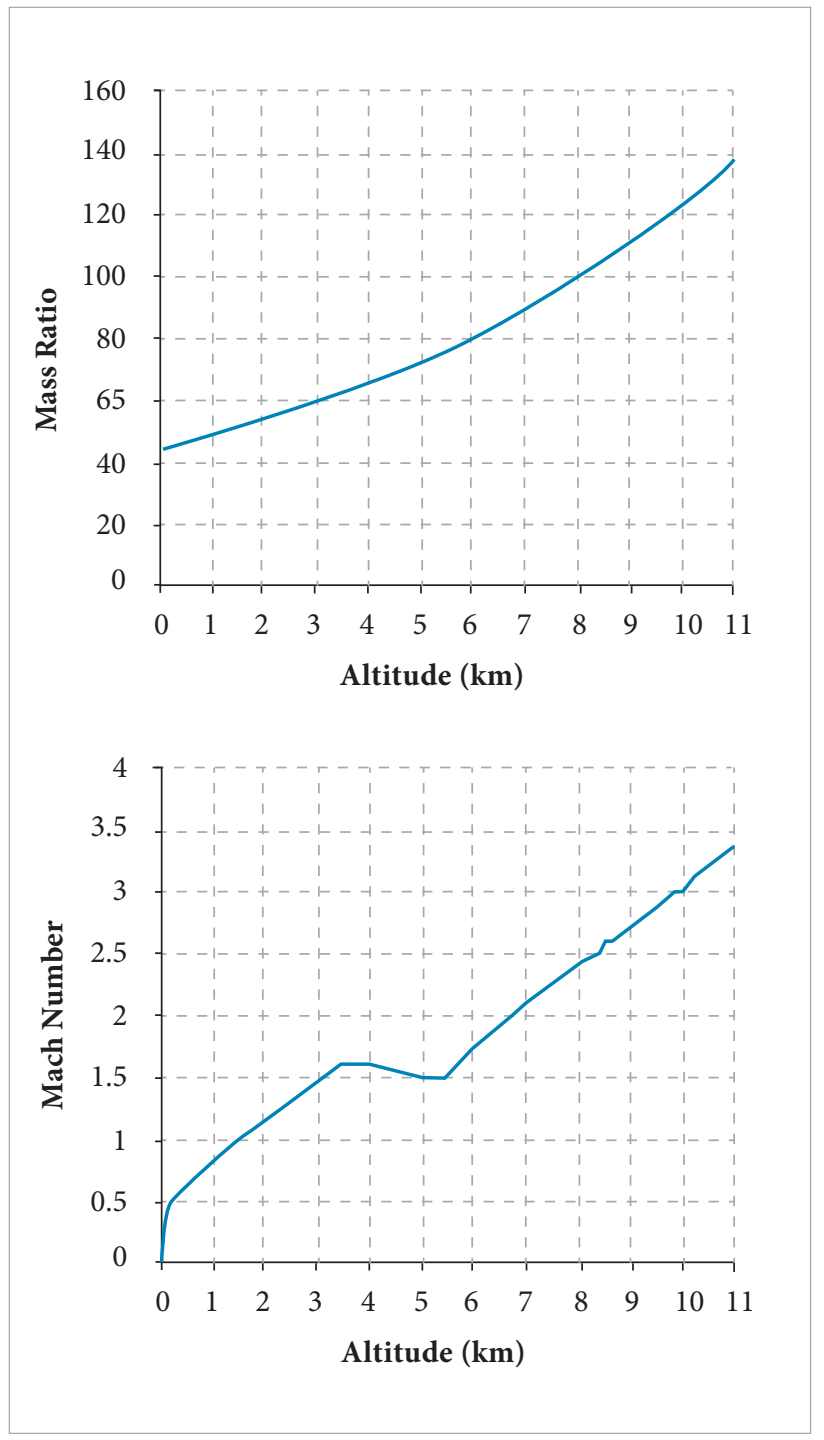

Figure 10. Mass ratio and Mach number as functions of the flight altitude. can be seen in the right side of Fig. 13. The coupling mechanism is altered in such a way that the flutter speed is lower with the increase of the roll rate. Such behavior occurs because the increase of the natural frequencies of the first fin antisymmetric bending mode is mostly subjected to differential stiffness effects in relation to the first fin anti-symmetric torsion mode. That is, the apparent increase in stiffness of the fin leads to an increase in the natural frequency. Therefore, there is a contribution for the coupling between the bending and torsion modes, while the frequency remains almost unaltered.

Another interesting behavior to be noted concerns a second and third coupling between the 6th and 8th, and 5th and 10th modes, respectively. The coalescence of these modes leads to higher flutter speeds, which are, therefore, not taken into account in the present analysis. However, the reader should observe that the spinning effects for such couplings are more significant than

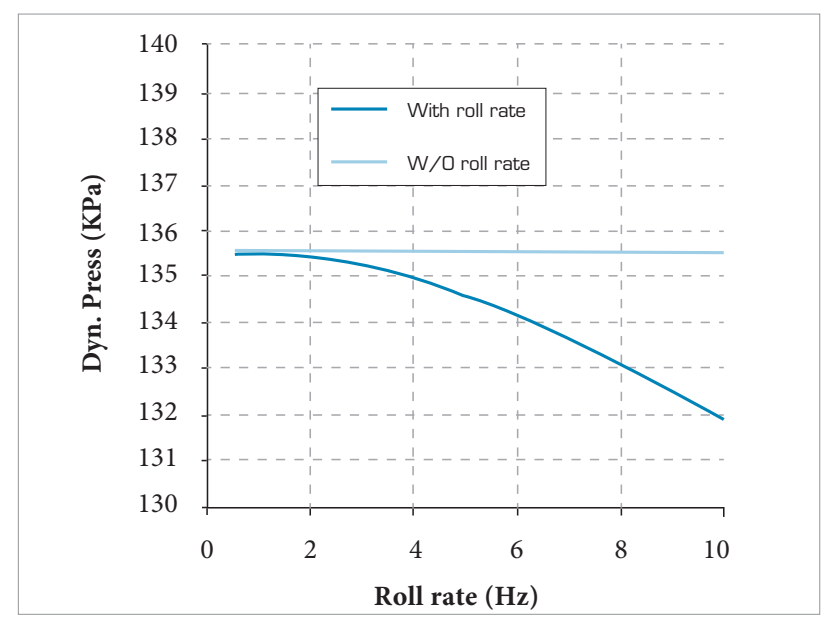

Figure 12. Dynamic pressure as a function of the vehicle roll rate.

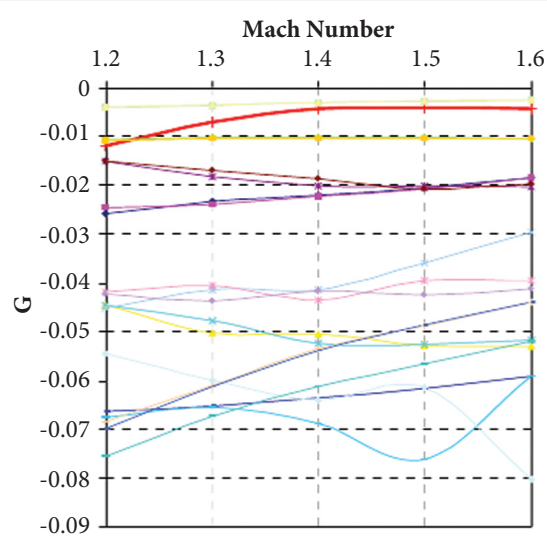

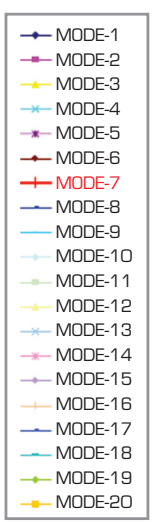

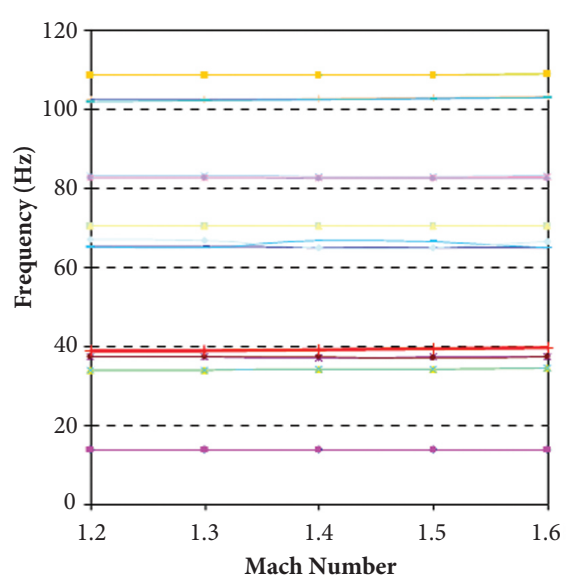

Figure 11. Matched point flutter analysis results for the two-module payload configuration. 

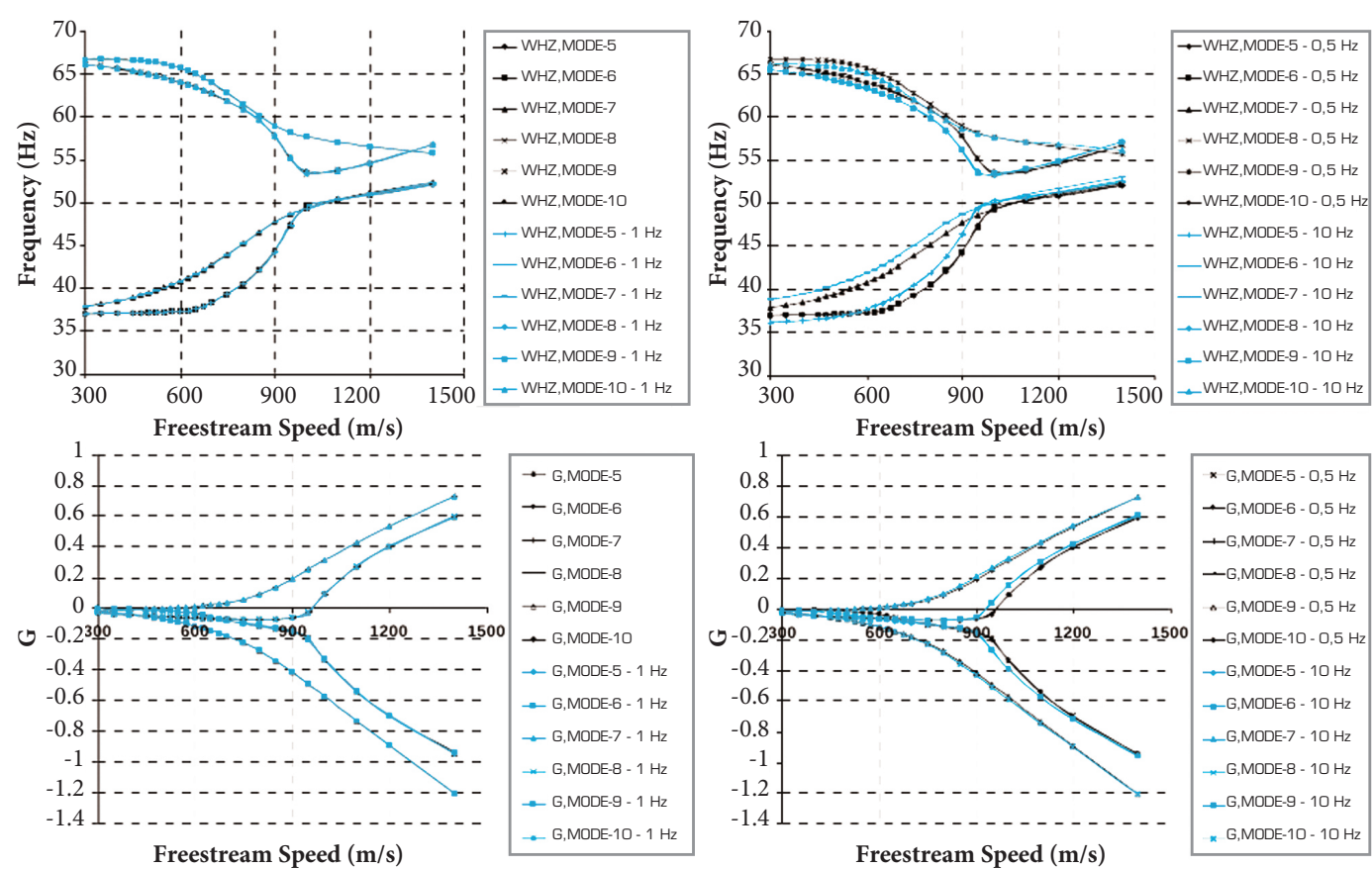

Figure 13. Evolution of the damping and frequencies as a function of the freestream speed.

the effects observed in the 7th-9th mode coupling. The reason for the augmented sensitivity due to increasing roll rates is associated to the types of mode shapes involved in the coupling. The modes, which primarily compose the associated flutter mechanisms, have an important contribution from the body displacement terms. Since body modes contribute to the coupling, the spin "softening effect" may also contribute for the early coalescence of the modes involved in the flutter mechanism.

\section{CONCLUSIONS AND RECOMMENDATIONS}

Results from the present investigation show the aeroelastic behavior of the VSB-30 sounding rocket regarding flutter. The present effort is the beginning of an investigation whose objectives are beyond the scope the aeroelastic analysis presented herein. Unlike conventional aeroelastic analysis of aircraft, aeroelastic stability of rockets depends mostly on the environmental conditions and operational aspects, such as the flight phase. The present study serves as a guideline for future enhancement on the aeroelastic analysis process and for the design of flight instrumentation for test-analysis correlations. As previously discussed, the vehicle is free from flutter throughout its flight envelope, considering reasonable flutter margins. However, the spinning effect is an influence to be considered in further investigations. In the present analyses, such effect was neglected due to vehicle low roll rates.

As expected, it was found that the smallest flutter margins occur at lower mass ratio conditions, when the flow is transonic. Therefore, further investigation of the VSB-30 transonic aeroelastic stability margins is strongly recommended, preferably using a higher fidelity aerodynamic formulation. Moreover, aeroelastic dynamic responses should also be explored, besides aeroelastic stability, in order to quantify the vibration levels of the vehicle and to correlate them with flight vibration data. Actually, as a continuation of the present investigation, a study of the correlation of the aeroelastic response with flight data would be extremely helpful. At transonic flow conditions, there are severe shock induced vibration characteristics which can compromise the operation of the scientific instrumentation at the payload modules.

Future refinements of the analyses here presented, in an attempt to further enhance the methodology for aeroelastic clearance of spinning sounding rockets, must include the effects of the gyroscopic stiffness and damping matrices. Aerodynamic model development for transonic flow 
conditions must also be included. The aerodynamic models for transonic flow should be based on field panel methods or linear lifting surface methods corrected for transonic flow conditions. These approaches are the most adequate when the aeroelastic analysis is included in a MDO process. Furthermore, computational aeroelasticity analyses, based on the solution of the non-linear fluid dynamic equations, could be considered for validation of the transonic correction methods previously mentioned. Finally, since the VSB-30 rocket could be used for reentry aerodynamics, aerothermoelasticity at hypersonic flow conditions may also be included in the design process. The linear aeroelastic model, or the one based on non-linear unsteady aerodynamics, could be employed for the aeroelastic stability and response analyses at reentry conditions.

\section{ACKNOWLEDGMENTS}

The authors would like to acknowledge Mr. Sidney Servulo Cunha Yamanaka, for providing the flight dynamic data of the VSB-30 sounding rocket. Partial support for this work was provided by Conselho Nacional de Desenvolvimento Científico e Tecnológico, CNPq, under Research Grants No. 312064/2006-3 and No. 471592/2011-0.

\section{REFERENCES}

Azevedo, J.L.F., 1988, "Transonic Aeroelastic Analysis of Launch Vehicle Configurations," NASA CR - 4186.

Bae, J.S., Kim, D.K., Shih, W.H., Lee, I. and Kim, S.H., "Nonlinear Aeroelastic Analysis of a Deployable Missile Control Fin," Journal of Spacecraft and Rockets, Vol. 41, No. 2, pp. 264-271.

Chae, S., 2004, "Effect of Follower Forces on Aeroelastic Stability of Flexible Structures," Ph.D. Thesis, School of Aerospace Engineering, Georgia Institute of Technology, Atlanta, GA.

Chae, S. and Hodges, D.H., 2003, "Dynamics and Aeroelastic Analysis of Missiles," 44th AIAA/ASME/ASCE/AHS Structures, Structural Dynamics, and Materials Conference, Norfolk, VA.

Chen, P.C., 1999, "A Damping Perturbation Method for Flutter Solution: The g-Method," 2nd AIAA/CEAS International Forum on Aeroelasticity and Structural Dynamics, Williamsburg, VA.

Chen, P.C. and Liu, D.D., 1990, "Unsteady Supersonic Computation for Arbitrary Wing-Body Configuration Including External Stores," Journal of Aircraft, Vol. 27, No. 2, pp. 108-116.

Duarte, J.A.A., Damilano J.G., Almeida, F.E. and Odaguiri, P.K., 2005, "Flight Data Used on the Evaluation of the Acceptance Testing Specifications for the VSB-30 Sounding Rocket," Proceedings of the 17th ESA Symposium, Sandefjord, Norway.

Francesco Capri, F., Mastroddi, F. and Pizzicaroli, A., 2006, "Linearized Aeroelastic Analysis for a Launch Vehicle in Transonic Flight Conditions," Journal of Spacecraft and Rockets, Vol. 43, No. 1, pp. 92-104.

Garcia-Fogeda, P. and Liu, D.D., 1988, "Supersonic Aeroelastic Applications of Harmonic Potential Panel Method to Oscillating Flexible Bodies," Journal of Spacecraft and Rockets, Vol. 25, No. 4, pp. 271-277.

Haddadpour, H., 2006, "Aeroservoelastic Stability of Supersonic SlenderBody Flight Vehicles," Journal of Guidance, Control and Dynamics, Vol. 29, No. 6, pp. 1423-1427.

Heddadj, S. and Cayzac, R., 2000, "Aeroelasticity of High L/D Supersonic Bodies: Theoretical and Numerical Approach," 38th AIAA Aerospace Sciences Meeting \& Exhibit, Reno, NV.

Liu, D.D., Yao, Z.X., Sarhaddi, D. and Chavez, F., 1997, "From Piston
Theory to a Unified Hypersonic-Supersonic Lifting Surface Method," Journal of Aircraft, Vol. 34, No. 3, pp. 304-312.

Livshits, D.S., Yaniv, S. and Karpel, M., 1996, "Dynamic Stability of Free Flight Rockets," 37th AIAA/ASME/ASCE/AHS/ASC Structures, Structural Dynamics, and Materials Conference and Exhibit, Salt Lake City, UT.

Martin, D.J., 1958, "Summary of Flutter Experiences as a Guide to the Preliminary Design of Lifting Surfaces on Missiles," NACA TN - 4197.

McNamara, J.J. and Friedmann, P.P., 2006, "Aeroelastic and Aerothermoelastic Analysis of Hypersonic Vehicles: Current Status and Future Trends," AIAA Paper No. 2006-8058, 14th AIAA/AHI Space Planes and Hypersonic Systems and Technologies Conference, Canberra, Australia.

Meyers, S.C., 1973, "Aeroelastic Analysis of Sounding Rocket Vehicles," AIAA Paper No. 73-284.

MSC/NASTRAN, 1995, Software Package User's Guide, V. 68.2, MacNeal-Schwendler Corporation, Los Angeles, CA.

MSC/NASTRAN V70, 1995, Advanced Dynamics User’s Guide, MacNealSchwendler Corporation, Los Angeles, CA.

Murphy, C.H. and Mermagen, W.H, 2001, "Flight Mechanics of an Elastic Symmetric Missile," Journal of Guidance, Control and Dynamics, Vol. 24, No. 6, pp. 1125-1132.

Murphy, C.H. and Mermagen, W.H., 2005, "Aero-Elastic Motion of a SpinStabilized Projectile," ARL-TR-3453, Aberdeen Proving Ground, MD.

Paek, S.K., Bae, J.S. and Lee, I., 2002, "Flutter Analysis of a Wraparound Fin Projectile Considering Rolling Motion," Journal of Spacecraft and Rockets, Vol. 39, No. 1, pp. 66-72.

Platus, D.H., 1992, "Aeroelastic Stability of Slender, Spinning Missiles," Journal of Guidance, Control and Dynamics, Vol. 15, No.1, pp. 144-151 .

Turner, J., Hörschgen, M., Jung, W., Stamminger, A. and Turner, P., 2006, "SHEFEX-Hypersonic Re-entry Flight Experiment Vehicle and Subsystem Design, Flight Performance and Prospects," AIAA Paper No. 2006-8115, 14th AIAA/AHI Space Planes and Hypersonic Systems and Technologies Conference, Canberra, Australia.

ZAERO, 2003, Software Package, Ver. 6.5, Zona Technology, Scottsdale, AZ. 